\title{
Introducing IST Austria
}

\section{László Erdős and Robert Seiringer}

A few years ago Klosterneuburg, a gemütlich small Austrian town near Vienna, was known for its magnificent 900-year-old monastery and the fertile vineyards on the slopes of the Vienna Woods. Since 2009 it has also been the location of a brand new scientific institute, a bold initiative of the Austrian government to foster research and graduate education in the natural sciences. While a large part of the new campus is still under construction and only about one third of the planned research groups are operational, it is already a vibrant intellectual community. Mathematics, as the common denominator of all quantitative sciences, is one of the key areas that anticipate a vigorous expansion in the next few years. In this article we present our new home, the Institute of Science and Technology Austria (IST Austria).

\section{The Mission of IST Austria}

IST Austria is a multidisciplinary research institution dedicated to cutting-edge basic research in the natural, mathematical, and computer sciences, both theoretical and experimental. It is not a university in the traditional sense; its focus is on curiosity-driven research and the training of graduate students. The long-term financial commitment from the Austrian federal government and from the state of Lower Austria, as well as the governance and management structures of IST Austria, guarantee the institute's freedom from political and commercial influences.

We believe IST Austria is almost unique in the world; its structure is probably closest to the Weizmann Institute. In fact, Professor Haim Harari, former president of the Weizmann Institute, has played a pivotal role in setting up the concept of IST Austria. The vision was to create a special environment where researchers from different disciplines have much more interaction than at a traditional institute that has separate faculties, departments, and schools.

László Erdós is professor at IST Austria. His email address is 1asz1o.erdos@ist.ac.at.

Robert Seiringer is professor at IST Austria. His email address is robert. seiringer@ist.ac . at.

DOI: http://dx.doi.org/10.1090/noti1202
To foster a creative and interdisciplinary scientific atmosphere, all hierarchical and separating organizational structures, such as departments, are avoided. The scientists are organized into independent research groups, each headed by a professor or a tenure-track assistant professor.

Jointly designed courses, interdisciplinary seminars, an institute-wide hiring process, and joint social activities bring together mathematicians, physicists, computer scientists, neuroscientists, and biologists. Interdisciplinary research is not required, as scientific excellence is the only criterion for evaluating our performance, but the open environment strongly encourages interactions. The goal is to foster a culture of openness and pass it on to our students. The official language on the whole campus is exclusively English.

\section{Facts and Figures}

IST Austria, inaugurated in 2009, was established jointly by the federal government of Austria and the provincial government of Lower Austria. The campus is $18 \mathrm{~km}$ from the center of Vienna; the subway is accessible by a special shuttle service as well as with public buses in twenty-five minutes. The first laboratory building was completed in 2010 and the second in 2012. Further buildings are under construction or in the planning phase; our campus can accommodate several more office and lab buildings as well as student housing, sport, and recreational facilities.

For the period from 2007 until 2026, the federal government of Austria will provide up to 1,280 million euros (US $\$ 1,750$ million) in operational funds. Two thirds of this budget is guaranteed, while the remaining third is conditional on performancerelated criteria such as the raising of third-party funds. The state of Lower Austria contributes the budget for construction and campus maintenance, in a total amount of 510 million euros (US $\$ 700$ million) from 2007 until 2026. By the end of 2013, IST Austria had obtained commitments for more than 36 million euros in research grants, among them fourteen European Research Council grants. We have also received 17 million euros in donations so far.

As of mid-2014, there were twenty-nine independent research groups on campus, with another 
four to start in the coming months. Independence is especially attractive for younger scientists in experimental areas; IST Austria gives them the opportunity to build their own labs. The development plan of IST Austria allows for a growth to ninety research groups by 2026; i.e., we plan to hire five to six new faculty members every year in the next decade.

\section{Faculty and Hiring}

To keep the hierarchy minimal, there are only two levels of professors. Younger scientists are hired as tenure-track assistant professors, and they can immediately form their research group and supervise postdocs and students. Their promotion to the level of professor with a permanent contract is similar to the tenure procedure at leading US institutions. Research excellence and promise are the exclusive hiring criteria for all scientists at IST Austria.

The institute's choice of scientific topics is based solely on the availability of outstanding individuals. The institute does not allocate a predetermined number of positions to different disciplines, and every faculty member participates in every hiring discussion in a very open style. Currently half of our faculty works in life sciences, followed by computer science as the second most represented area. Mathematics and physics still lag behind, but concentrated efforts in the last two years have improved the balance.

\section{Mathematics at IST Austria}

The very first professor on campus was Nick Barton, an eminent British evolutionary biologist with strong connections to mathematics. He was followed by Herbert Edelsbrunner, a very broad Austrian mathematician and computer scientist working in computational geometry and topology. Several new mathematical directions have been established by hiring younger colleagues (Caroline Uhler, mathematical statistics; Uli Wagner, combinatorial geometry) in addition to computer scientists working in cryptography, machine learning, algorithms, and computational fluid dynamics. With the recent hiring of Robert Seiringer (quantum statistical mechanics) and László Erdős (random matrices and disordered systems), mathematical physics and analysis in a broader sense were established. A natural link between the discrete and continuous mathematics is our newest colleague, Jan Maas, working on discrete optimal transportation problems.

While our hiring concept is open to every direction in mathematics and its applications, we strive for a healthy balance and hope to hire colleagues in other key directions such as partial differential equations, probability theory, geometry, or algebra. We envision a development plan where every new colleague feels connected to at least one existing group without duplicating it.

Our mathematics-oriented faculty is well embedded in the interdisciplinary environment of IST Austria via their natural links to biology, computer science, and physics. While an interdisciplinary attitude is always welcome, we fully recognize that several core areas of mathematics do not have a direct interdisciplinary component. In order to promote integrity of mathematics on campus and bring IST Austria onto the world map of the mathematics community, we keep our doors open to excellent mathematicians working in such areas as well.

\section{Opportunities}

IST Austria is constantly seeking applications for full professor positions from experienced scientists with high international reputations. Every fall we also have a call for applications on the tenure-track level; a typical applicant is $3-8$ years after the $\mathrm{PhD}$ and already has an outstanding record of independent research. Since hiring is done on an institute-wide level, attracting many excellent candidates in mathematics is a key to strengthening its presence at IST Austria.

For scientists with fresh PhDs, IST Austria has a centrally financed postdoctoral program, the IST Fellowship. Applications are solicited, with deadlines of March 15 and September 15. The selection is done by a single committee; hence candidates from different disciplines compete.

Our graduate school follows the US admission pattern: we admit students once a year; the application deadline is January 15. Currently we admit about thirty students per year, but the size of the graduate class will increase as IST Austria expands. Students are provided with a generous fellowship for the whole duration of their studies, assuming satisfactory progress.

We have a long-term visitor program with partial financing designed for experienced scientists spending a 3- to 12-month period at IST Austria; this is an opportunity for people on sabbatical.

Finally, we offer an internship program (called ISTernship) for talented and motivated undergraduate students who wish to spend 8-12 weeks during the summer at IST Austria.

In addition to the central programs, every research group has its own research budget (partly allocated from the central budget, partly from third-party fundings). At the discretion of the group leader, this budget can also be used to hire postdocs or to invite visitors.

A more detailed description of these opportunities may be found on our website www.ist. ac.at. 